\title{
A NOTE ON THE THREE-STAGE GROWTH MODEL
}

\author{
SVETOSLAV MARKOV ${ }^{1}$, ANTON ILIEV $^{2}$, \\ ASEN RAHNEV ${ }^{3}$, AND NIKOLAY KYURKCHIEV ${ }^{4}$ \\ ${ }^{1}$ Institute of Mathematics and Informatics \\ Bulgarian Academy of Sciences \\ Acad. G. Bonchev Str., Bl. 8, 1113 Sofia, BULGARIA \\ ${ }^{2,3,4}$ Faculty of Mathematics and Informatics \\ University of Plovdiv Paisii Hilendarski \\ 24, Tzar Asen Str., 4000 Plovdiv, BULGARIA
}

\begin{abstract}
In this paper we study the one-sided Hausdorff approximation of the generalized cut function by sigmoidal modified three-stage growth model. The model has a certain right of existence insofar as the theory of sigmoidal functions is well developed. The estimates of the value of the best Hausdorff approximation obtained in this article can be used in practice as one possible additional criterion in "saturation" study. We examine the small data for modeling the growth of red abalone Haliotis Rufescens in Northern California. Numerical examples are presented using CAS MATHEMATICA.
\end{abstract}

AMS Subject Classification: $41 \mathrm{~A} 46$

Key Words: modified three-stage growth model, generalized cut function associated to the model, Hausdorff distance, upper and lower bounds

Received: $\quad$ May 10, 2018; Revised: November 1, 2018;

Published (online): November 9, $2018 \quad$ doi: $\quad 10.12732 /$ dsa.v28i1.4

Dynamic Publishers, Inc., Acad. Publishers, Ltd. https://acadsol.eu/dsa

\section{INTRODUCTION}

Let us examine the following three-stage growth model

$$
A \stackrel{k_{1}}{\longrightarrow} B \stackrel{k_{2}}{\longrightarrow} C
$$




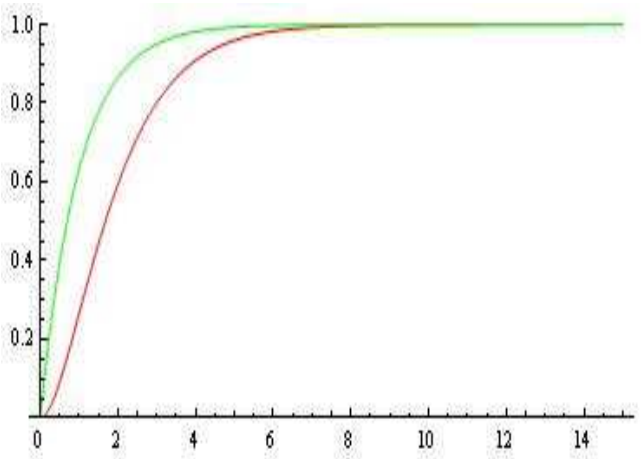

Figure 1: Three-stage growth model $\tilde{C}(t)$ (sigmoidal; red) for $n=k_{2}-k_{1}$, $k_{1}=1, k_{2}=1.001$ and three-stage model $C(t)$ (first order; green) for $n=$ $k_{1}=k_{2}, k_{1}=1, k_{2}=2500$.

with two steps $\left(k_{1}\right.$ and $\left.k_{2}\right)$ depending on the ratio of the growth parameters $\frac{k_{1}}{k_{2}}$.

For the mechanism the following system of ODEs is known [1]:

$$
\begin{aligned}
& d A(t) / d t=-k_{1} A(t), \\
& d B(t) / d t=k_{1} A(t)-k_{2} B(t), \\
& d C(t) / d t=k_{2} B(t), \\
& A(0) \quad A_{0}, \quad B(0)=0, \quad C(0)=0 .
\end{aligned}
$$

Noticing that $d A / d t+d B / d t+d C / d t=0$, hence $A+B+C=A_{0}$, and at any time, we find

$$
C(t)=A_{0}-B(t)-A(t)
$$

or $[4]$ :

$$
C(t)=A_{0}\left(1-\frac{k_{1}}{k_{1}-k_{2}}\left(e^{-k_{1} t}-e^{-k_{2} t}\right)-e^{-k_{1} t}\right) .
$$

For some details, see [2], [3]. In [4], the authors debated to the following modified model for the individual growth of marine invertebrates:

$$
\tilde{C}(t)=A_{0}\left(1-\frac{k_{1}}{n}\left(e^{-k_{1} t}-e^{-k_{2} t}\right)-e^{-k_{2} t}\right)
$$

where $n=k_{2}-k_{1}$, and $\frac{k_{1}}{k_{2}}$ is close to 1 .

The model $\tilde{C}$ predicts sigmoidal growth (see, Figure 1), i.e. in a three-stage growth model, the shape is controlled by the ratio $\frac{k_{1}}{k_{2}}$.

For $3 D$-surface plot for the three-stage mechanism in the range $n=k_{2}-k_{1}$, or $n=k_{1}-k_{2}$, see, Figure 2 . 

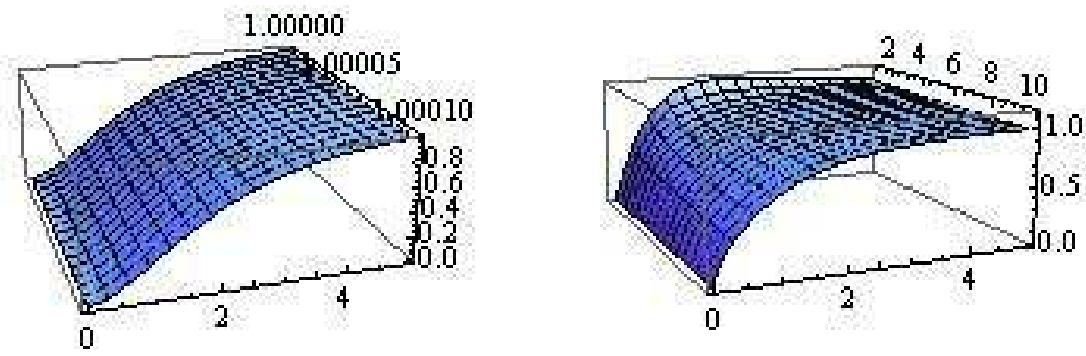

Figure 2: $3 D$-surface plot for the three-stage mechanism in the range $n=$ $k_{2}-k_{1}$, or $n=k_{1}-k_{2}$.

\section{MAIN RESULTS}

Without loosing of generality, for $A_{0}=1$ and $n=k_{2}-k_{1}>0, \frac{k_{1}}{k_{2}} \rightarrow 1$ we consider the following family:

$$
\tilde{C}(t)=1-\frac{k_{1}}{n}\left(e^{-k_{1} t}-e^{-k_{2} t}\right)-e^{-k_{2} t} .
$$

We find that the sigmoid (1) has an inflection at point:

$$
t^{*}=\frac{1}{n} \ln \left(\frac{\left(-k_{2}^{2}+\frac{k_{1} k_{2}^{2}}{n}\right) n}{k_{1}^{3}}\right) .
$$

Definition 1. The associate to the (1) cut function $\tilde{C}^{*}$ is defined by

$$
\tilde{C}^{*}(t)=\left\{\begin{array}{cl}
0, & \text { if } t<t_{1}, \\
\tilde{C}^{\prime}\left(t^{*}\right)\left(t-t^{*}\right)+\tilde{C}\left(t^{*}\right), & \text { if } t_{1} \leq t<t_{2}, \\
1, & \text { if } t \geq t_{2} .
\end{array}\right.
$$

The straight line $y=\tilde{C}^{\prime}\left(t^{*}\right)\left(t-t^{*}\right)+\tilde{C}\left(t^{*}\right)$ cross the lines $y=0$ and $y=1$ at the points $t_{1}$ and $t_{2}$.

Definition 2. [6] The one-sided Hausdorff distance $\vec{\rho}(f, g)$ between two interval functions $f, g$ on $\Omega \subseteq \mathbb{R}$, is the one-sided Hausdorff distance between their completed graphs $\mathcal{F}(f)$ and $\mathcal{F}(g)$ considered as closed subsets of $\Omega \times \mathbb{R}$. More precisely,

$$
\vec{\rho}(f, g)=\sup _{B \in \mathcal{F}(g)} \inf _{A \in \mathcal{F}(f)}\|A-B\|
$$

where $\|\cdot\|$ is a norm in $\mathbb{R}^{2}$. 
We recall that completed graph of $f$ is the closure of the graph of $f$ as a subset of $\Omega \times \mathbb{R}$. If the graph of an interval function $f$ equals $\mathcal{F}(f)$, then the $f$ is called S-continuous.

The Hausdorff distance $\rho(f, g)=\max \{\vec{\rho}(f, g), \vec{\rho}(g, f)\}$ defines a metric in the set of the S-continuous interval functions [7]-[10].

\subsection{APPROXIMATION OF THE CUT FUNCTION (2) BY SIGMOID FUNCTION (1)}

The one-sided Hausdorff distance $d$ between the functions (1) and (2) satisfies the relation

$$
\tilde{C}\left(t_{2}+d\right)=1-d
$$

The following theorem gives upper and lower bounds for $d$

Theorem 1. Let

$$
\begin{gathered}
p=-e^{-k_{2} t_{2}}-\frac{k_{1}}{n} e^{-k_{1} t_{2}}+\frac{k_{1}}{n} e^{-k_{2} t_{2}}, \\
q=1+k_{2} e^{-k_{2} t_{2}}+\frac{k_{1}^{2}}{n} e^{-k_{1} t_{2}}-\frac{k_{1} k_{2}}{n} e^{-k_{2} t_{2}} \\
r=-2 \frac{q}{p} ; n=k_{2}-k_{1}>0 ; \frac{k_{1}}{k_{2}} \rightarrow 1 ; \frac{2 k_{1}-k_{2}}{k_{1}}<e^{t_{2}\left(k_{2}-k_{1}\right)} .
\end{gathered}
$$

For the one-sided Hausdorff distance $d$ between $\tilde{C}^{*}(t)$ and the sigmoidal function (1) the following inequalities hold for: $r>e^{2}$

$$
d_{l}=\frac{1}{r}<d<\frac{\ln r}{r}=d_{r}
$$

Proof. Let us examine the function:

$$
F(d)=\tilde{C}\left(t_{2}+d\right)-1+d
$$

From $F^{\prime}(d)>0$ we conclude that function $F$ is increasing.

Consider the function

$$
G(d)=p+q d
$$

From Taylor expansion we obtain $G(d)-F(d)=O\left(d^{2}\right)$.

Hence $G(d)$ approximates $F(d)$ with $d \rightarrow 0$ as $O\left(d^{2}\right)$ (see Figure 3).

In addition $G^{\prime}(d)>0$.

From the conditions of the theorem, we see that $p<0$ and $q>0$ and $G\left(d_{l}\right)=$ $\frac{1}{2} p<0$. 


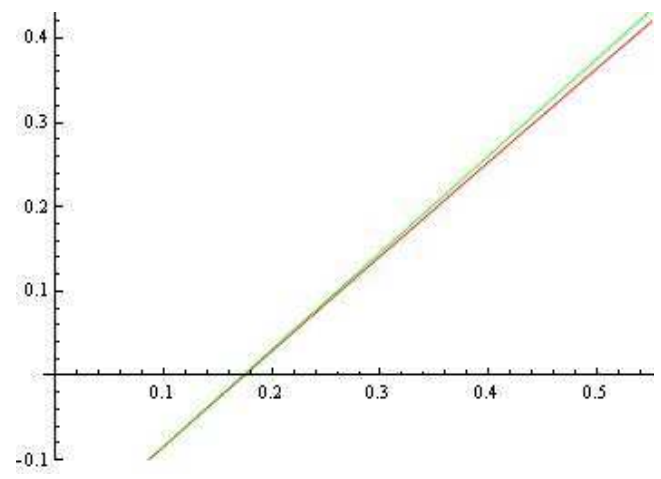

Figure 3: The functions $F(d)$ and $G(d)$ for $k_{1}=1 ; k_{2}=1.01$.

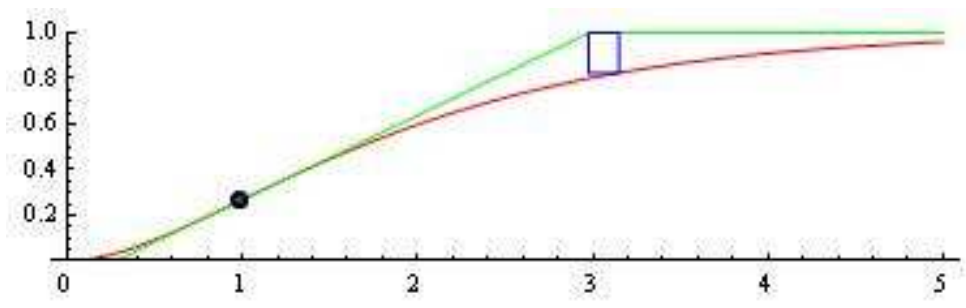

Figure 4: The cut function $\tilde{C}^{*}(t)$ and the sigmoidal function $\tilde{C}(t)$ with $k_{1}=1$, $k_{2}=1.01, t^{*}=0.985033, t_{1}=0.27045, t_{2}=2.97525 ; \mathrm{H}-$ distance $d=$ $0.174444, d_{l}=0.0865764, d_{r}=0.211829$.

Further, for $r>e^{2}$ we have $G\left(d_{r}\right)>0$.

This completes the proof of the theorem.

The model (1) for $k_{1}=1, k_{2}=1.01, t^{*}=0.985033, t_{1}=0.27045, t_{2}=2.97525$ is visualized on Figure 4.

From the nonlinear equation (3) and inequalities (4) we have: $d=0.174444$, $d_{l}=0.0865764, d_{r}=0.211829$.

\subsection{NUMERICAL EXAMPLE}

We examine the following data. (The small data for modeling the growth of red abalone is shown in Table 1. For more details, see [5]). 


\begin{tabular}{|c|c|}
\hline Age & Length $(\mathrm{mm})$ \\
\hline 1 & 16.1 \\
2 & 33.9 \\
3 & 54.3 \\
4 & 76.2 \\
5 & 97.8 \\
6 & 117.1 \\
7 & 133.3 \\
8 & 146.5 \\
9 & 157.2 \\
10 & 166 \\
11 & 173.3 \\
12 & 179.6 \\
\hline
\end{tabular}

Table 1: The small data for modeling the growth of red abalone Haliotis Rufescens in Northern California [5]

The model (2) based on the data of Table 1 for the estimated parameters:

$$
A_{0}=179.6 ; \quad k_{2}=0.4384 ; \quad k_{1}=0.434133 ; t^{*}=2.26955 ; t_{1}=0.62341 ; t_{2}=6.854
$$

is plotted on Figure 5 .

Specifically, we will note that the growth model functions is checked by an additional six criteria, the consideration of which go beyond this article.

For example, for the predictive power (PP) criterion

$$
P P=\sum_{i=1}^{n}\left(\frac{\tilde{C}\left(t_{i}\right)-y_{i}}{y_{i}}\right)^{2}
$$

measures the distance of model actual data from the estimates against the actual data, we find $P P=0.243413$.

Remarks. The model $\tilde{C}(t)$ has a certain right of existence insofar as the theory of sigmoidal functions is well developed.

Of course, its use in approximating such data is associated with a loss of accuracy when using the operator of the programming environment (for example, CAS Mathematica) to find a local extreme.

The estimates of the value of the best Hausdorff approximation obtained in this article can be used in practice as one possible additional criterion in "saturation" study. 


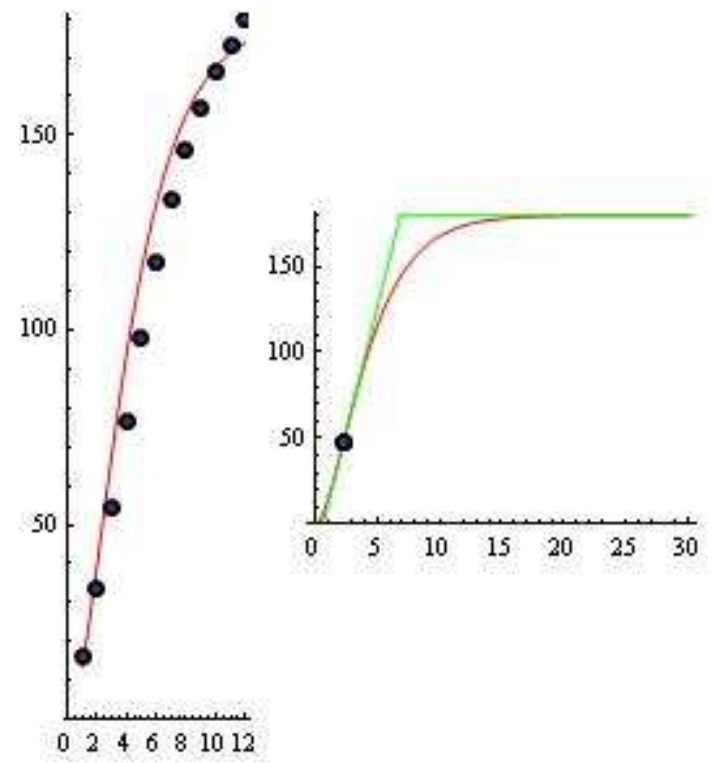

Figure 5: The model $\tilde{C}(t)$

For some approximation, computational and modelling aspects, see [11]-[32].

The results obtained in this paper can be used when controlling growth in Software Reliability Models, see [33], [34].

\section{ACKNOWLEDGMENTS}

This work has been supported by the project FP17-FMI-008 of Department for Scientific Research, Paisii Hilendarski University of Plovdiv.

\section{REFERENCES}

[1] A. Cornish-Bowden, Fundamentals of Enzyme Kinetics, Wiley-Blackwell (2012), ISBN: 978-3-527-33074-4.

[2] D. Soete, R. Gijbels, J. Hoste, Neuron Activation Analysis, Wiley-Interscience (1972).

[3] H. Bateman, The solution of a system of differential equations occurring in the theory of radio-active transformations, Proc. Cambridge Phil. Soc., 15 (1910), 423-427. 
[4] L. Rogers-Bennett, D. W. Rogers, A two-step growth curve: Approach to the von Bertalanffy and Gompertz equations, Adv. in Pure Math., 6 (2016), 321-330.

[5] L. Rogers-Bennett, D. W. Rogers, S. A. Schultz, Modeling growth and mortality of red abalone Haliotis Rufescens in Northern California, J. of Shellfish Research, 26 No. 3 (2007), 719-727.

[6] B. Sendov, Hausdorff Approximations, Kluwer, Boston (1990).

[7] R. Anguelov, S. Markov, Hausdorff Continuous Interval Functions and Approximations, In: SCAN 2014 Proceedings, LNCS, ed. by J.W.von Gudenberg, Springer, Berlin (2015).

[8] R. Anguelov, S. Markov, B. Sendov, On the Normed Linear Space of Hausdorff Continuous Functions. In: Lirkov, I., et al. (Eds.): Lecture Notes in Computer Science, 3743, Springer (2006), 281-288.

[9] R. Anguelov, S. Markov, B. Sendov, Algebraic Operations on the Space of Hausdorff Continuous Functions. In: Bojanov, B. (Ed.): Constructive Theory of Functions, Prof. M. Drinov Academic Publ. House, Sofia (2006), 35-44.

[10] R. Anguelov, S. Markov, B. Sendov, The Set of Hausdorff Continuous Functions - the Largest Linear Space of Interval Functions, Reliable Computing, 12 (2006), $337-363$.

[11] N. Kyurkchiev, S. Markov, On the Hausdorff distance between the Heaviside step function and Verhulst logistic function, J. Math. Chem., 54 No. 1 (2016), 109-119.

[12] N. Kyurkchiev, S. Markov, Sigmoid functions: Some Approximation and Modelling Aspects, LAP LAMBERT Academic Publishing, Saarbrucken (2015), ISBN 978-3-659-76045-7.

[13] N. Kyurkchiev, A. Iliev, S. Markov, Some Techniques for Recurrence Generating of Activation Functions: Some Modeling and Approximation Aspects, LAP LAMBERT Academic Publishing (2017), ISBN: 978-3-330-33143-3.

[14] R. Anguelov, M. Borisov, A. Iliev, N. Kyurkchiev, S. Markov, On the chemical meaning of some growth models possessing Gompertzian-type property, Math. Meth. Appl. Sci., (2017), 1-12, doi:10.1002/mma.4539.

[15] R. Anguelov, N. Kyurkchiev, S. Markov, Some properties of the Blumberg's hyper-log-logistic curve, BIOMATH, 7 No. 1 (2018), 8 pp.

[16] A. Iliev, N. Kyurkchiev, S. Markov, On the Approximation of the step function by some sigmoid functions, Mathematics and Computers in Simulation, 133 (2017), $223-234$.

[17] A. Iliev, N. Kyurkchiev, S. Markov, Approximation of the cut function by Stannard and Richards sigmoid functions, IJPAM, 109 No. 1 (2016), 119-128. 
[18] S. Markov, A. Iliev, A. Rahnev, N. Kyurkchiev, A note on the Log-logistic and transmuted Log-logistic models. Some applications, Dynamic Systems and Applications, 27 No. 3 (2018), 593-607.

[19] S. Markov, N. Kyurkchiev, A. Iliev, A. Rahnev, On the approximation of the cut functions by hyper-log-logistic function, Neural, Parallel and Scientific Computations, 26 No. 2 (2018), 169-182.

[20] N. Kyurkchiev, A. Iliev, S. Markov, Families of recurrence generated three and four parametric activation functions, Int. J. Sci. Res. and Development, 4 No. 12 (2017), 746-750.

[21] N. Kyurkchiev, A note on the new geometric representation for the parameters in the fibril elongation process. C. R. Acad. Bulg. Sci., 69 No. 8, (2016), 963-972.

[22] N. Kyurkchiev, On the numerical solution of the general "ligand-gated neuroreceptors model' via CAS Mathematica, Pliska Stud. Math. Bulgar., 26 (2016), 133-142.

[23] N. Kyurkchiev, S. Markov, On the numerical solution of the general kinetic "Kangle" reaction system, Journal of Mathematical Chemistry, 54 No. 3 (2016), 792-805.

[24] L. Rogers-Bennett, D. Rogers, W. Bennett, T. Ebert, Modeling Red Sea Urchin Growth Using Six Growth Models, Fishery Bulletin, 101 (2003), 614-626.

[25] R. Leaf, L. Rogers-Bennett, Y. Jiao, Exploring the Use of a Size Based Egg per Recruit Model for the Red Abalone Fishery in California, North American Journal of Fisheries Management, 28 (2008), 1638-1647.

[26] N. Lester, B. Shuter, P. Abrams, Interpreting the von Bertalanffy Model of Somatic Growth in Fishes: The Cost of Reproduction, Proceedings of the Royal Society B: Biological Sciences, 271 (2004), 1625-1631.

[27] A. Hernandez-Llamas, D. Ratkowsky, Growth of Fishes, Crustaceans and Mollusks: Estimation of the von Bertalanffy, Logistic, Gompertz and Richards Curves and a New Growth Model, Marine Ecology Progress Series, 282 (2004), 237-244.

[28] L. von Bertalanffy, A Quantitative Theory of Organic Growth (Inquiries on Growth Laws. II), Human Biology, 10 (1938), 181-213.

[29] K. Sainsbury, Effect of Individual Variability on the von Bertalanffy Growth Equation, Canadian Journal of Fisheries and Aquatic Sciences, 37 (1980), 241247.

[30] P. Haaker, D. Parker, K. Barsky, C. Chun, Growth of Red Abalone, Haliotis rufescens (Swainson), at Johnson's Lee Santa Rosa Island, California, Journal of Shellfish Research, 17 (1998), 747-753. 
[31] N. Kyurkchiev, A. Iliev, Extension of Gompertz-type Equation in Modern Science: 240 Anniversary of the birth of B. Gompertz, LAP LAMBERT Academic Publishing (2018), ISBN: 978-613-9-90569-0.

[32] S. Markov, N. Kyurkchiev, A. Iliev, A. Rahnev, On the approximation of the generalized cut functions of degree $p+1$ by smooth hyper-log-logistic function, Dynamic Systems and Applications, 27 No. 4 (2018), 715-728.

[33] N. Pavlov, A. Iliev, A. Rahnev, N. Kyurkchiev, Some software reliability models: Approximation and modeling aspects, LAP LAMBERT Academic Publishing (2018), ISBN: 978-613-9-82805-0.

[34] N. Pavlov, A. Iliev, A. Rahnev, N. Kyurkchiev, Nontrivial Models in Debugging Theory (Part 2), LAP LAMBERT Academic Publishing (2018), ISBN: 978-6139-87794-2. 